\title{
El feedback entre iguales como medida de evaluación formativa para la mejora de la autorregulación del aprendizaje en la docencia virtual.
}

Fernando Senar Morera $^{a}$, Ursula Hinostroza Castillo ${ }^{\mathrm{b}}$, Adelina Ianos ${ }^{\mathrm{c}}$, Judit Janés Carulla ${ }^{\mathrm{d}}$, Cecilio Lapresta Rey ${ }^{\mathrm{e}}$, Rosa Florensa Guiuf, Ángel Huguet Canalís ${ }^{\mathrm{g}}$, Isabel Sáenz Hernández ${ }^{\mathrm{h}}$, Anna Casanovas Llorens ${ }^{\mathrm{i}}$ y Jordi Tarragona Foradada ${ }^{\mathrm{j}}$.

afernando.senar@udl.cat bursula.hinostroza@udl.cat cadelina.ianos@udl.cat djudit.janes@udl.cat

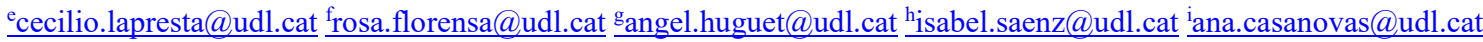
jitarragona@gss.scs.es

\section{\$EWWDWW}

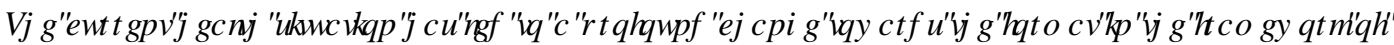

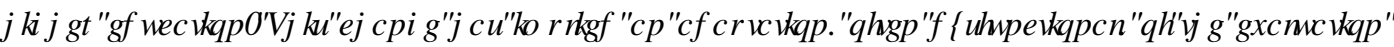

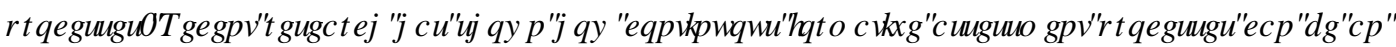

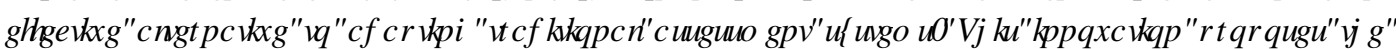

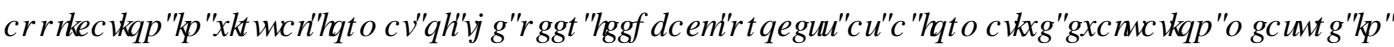

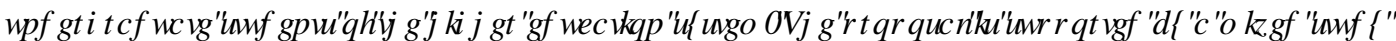

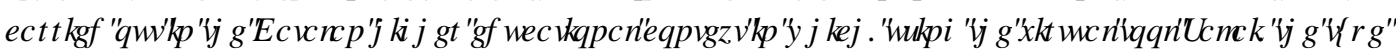

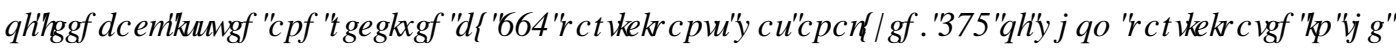

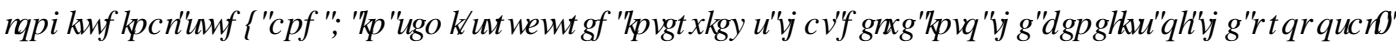

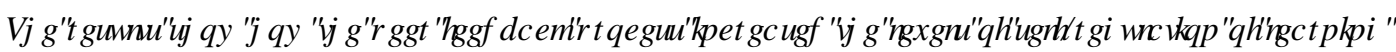

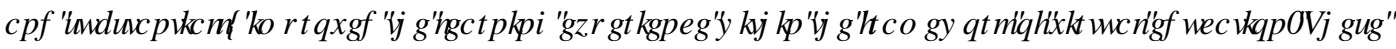

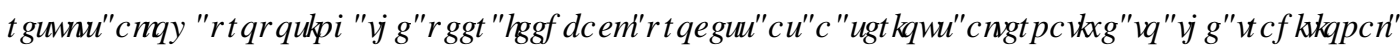

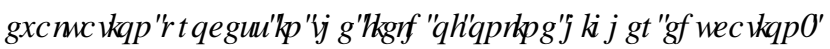

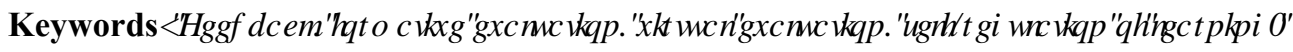

\section{HXP HQ}

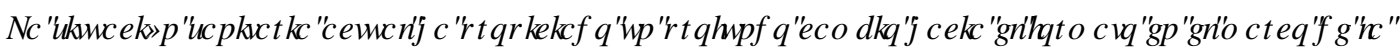

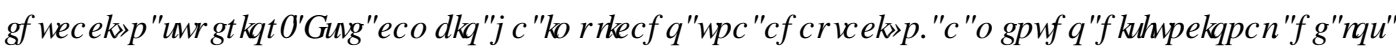

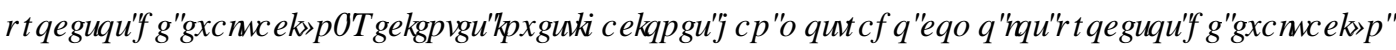

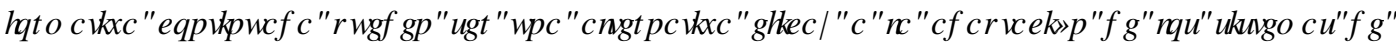

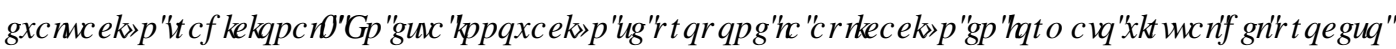

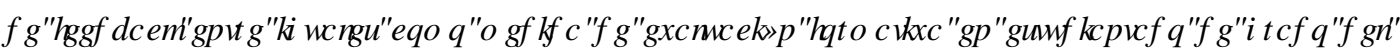

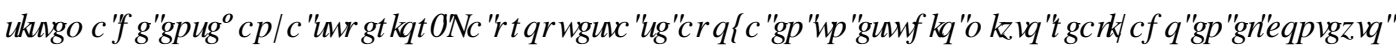

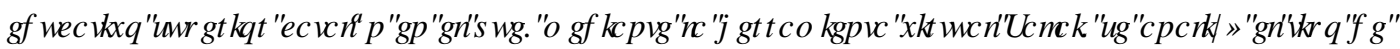

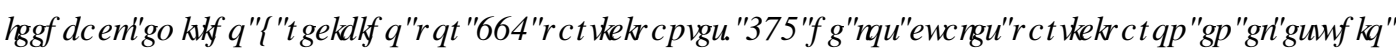

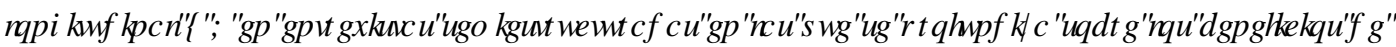

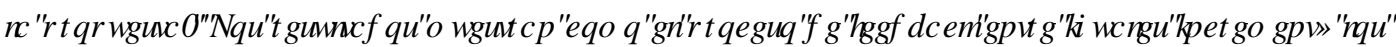

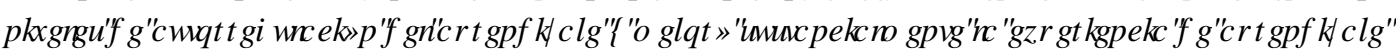

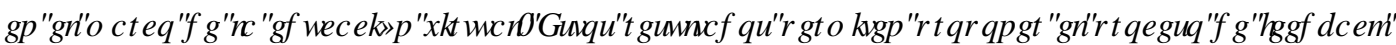

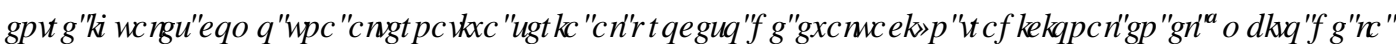
HEXFDFIYQUSHURURQOQHD 


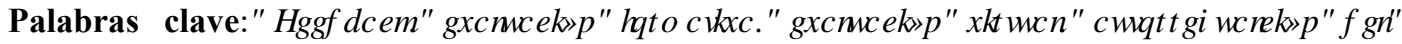 DSUHQD DNAIIII}

\section{Introducción}

La reestructuración del proceso de enseñanza-aprendizaje experimentada en el marco de la educación superior como consecuencia de la crisis sanitaria actual han supuesto un reto para la adaptación del proceso de evaluación. El paso de formato presencial a online ha hecho que los docentes se planteen la eficacia de la evaluación tradicional debido a la dificultad de asegurar la identidad de la persona que se somete a la prueba, así como controlar el contexto físico en el que se realiza dicha prueba (García Peñalvo et al., 2021). A su vez, elementos como la evaluación continuada, el uso de diferentes evidencias evaluativas y la habilitación de un sistema de evaluación entre pares han mostrado ser recursos eficaces en el formato de evaluación online (García Peñalvo et al., 2020). De este modo, la evaluación orientada al aprendizaje (Learning-oriented assessment, LOA), entendida como un enfoque particular de la evaluación formativa (Zeng et al, 2018) se ha convertido en una alternativa de evaluación eficaz ante el reto de la enseñanza online (Gibbons y Kankkonen, 2011).

Desde hace varias décadas, la evaluación formativa ha captado el interés de quienes estudian el proceso de enseñanza-aprendizaje. Su uso, tanto sustitutivo como complementario a las formas de evaluación acreditativa, permite que tanto docentes como estudiantes reciban información constante sobre el estado del aprendizaje y generen cambios en el mismo con el fin de poder optimizarlo (William, 2011). De esta manera, la evaluación formativa permite al docente ofrecer al alumnado una formación integral y holística (Bonsón y Águeda, 2005; Watts y García, 2006).

En el marco de la educación superior, el proceso de evaluación y feedback entre iguales es una de las propuestas de LOA que más atención ha recibido debido a su alto índice de éxito (véase, por ejemplo, Ajjawi y Boud, 2017; Coll et al., 2013). El feedback entre iguales, definido como un proceso de retroalimentación proporcionado por estudiantes con el mismo estatus, tiene como objetivo implicar al estudiante en el proceso de evaluación y, de esta forma, ser autónomo en la gestión de su propio aprendizaje. En otras palabras, busca la personalización del aprendizaje (Coll, 2016).

Diferentes estudios han observado como el proceso de feedback entre iguales ejerce un efecto positivo en diferentes aspectos del proceso de aprendizaje. Por ejemplo, el estudio de Cano y Pons (2019) muestra como el uso de esta estrategia facilita que el alumno encuentre sentido a la experiencia y que demuestre sus conocimientos, favoreciendo así aspectos cognitivos como la atención, la comprensión y la elaboración, y metacognitivos como la dirección, la evaluación y la modificación del propio aprendizaje. En lo relativo a habilidades sociales y comunicativas, los estudios de Carless y Chan (2017) y Neugebauer et al. (2016) muestran que necesidades como la de ofrecer críticas, justificar posiciones y tomar decisiones, inherentes a esta práctica, permite a los alumnos entrenar competencias como el trabajo en equipo y la negociación. Por último, el estudio de Ibarra Sáiz et al. (2012) destaca la contribución de esta práctica en la formación de competencias profesionales futuras al fomentar el pensamiento reflexivo, crítico e independiente. Además, dada la coyuntura actual, el feedback entre iguales es capaz de mejorar la experiencia universitaria del alumnado al aportar un sentimiento de comunidad que se ha visto afectado por la virtualidad (Caballé et al., 2011).

Por tanto, la presente innovación propone el proceso de feedback entre iguales como medida de evaluación formativa en el marco de la educación superior en un contexto de docencia virtual. La propuesta ha sido puesta en práctica universitario catalán en el que las restricciones derivadas de la crisis sanitaria han provocado el cambio de modalidad presencial a virtual. 


\section{Objetivos}

\section{Objetivo principal}

Analizar el feedback entre iguales en formato online como medida de evaluación formativa y su impacto en el proceso de autorregulación del aprendizaje.

\section{Objetivos específicos}

Conocer la eficacia del feedback entre iguales en la autorregulación del aprendizaje.

Identificar el tipo de feedback elaborado por el alumnado.

Analizar la eficacia del feedback entre iguales como herramienta para la mejora del aprendizaje virtual

\section{Desarrollo de la innovación}

Este estudio analizó el feedback de 442 alumnos pertenecientes a 11 grupos de 8 grados (educación social, educación infantil, educación primaria, doble titulación educación infantil y educación primaria, doble grado en educación primaria y ciencias de la actividad física y del deporte, administración y dirección de empresas, enfermería y psicología) en 7 asignaturas diferentes. De esta muestra, 153 alumnos realizaron un cuestionario de autorregulación administrado en dos tiempos (pre y post-test). Para conocer en profundidad la experiencia de aprendizaje de este alumnado se realizaron 9 entrevistas semiestructuradas.

Para la aplicación de la innovación se seleccionó una evidencia de evaluación de cada una de las asignaturas elegidas para el estudio. Debido a las diferencias metodológicas propias de cada grado, las evidencias seleccionadas son diferentes en cada asignatura. Sin embargo, la administración de la innovación siguió una pauta de actuación comunes en todas las asignaturas que se dividió en 10 fases, considerando las pautas propuestas por Cano et al. (2020). Todas las fases, excepto la primera, se realizaron a través de la herramienta virtual Sakai y de forma grupal:

3 UP HDIDMH Construir junto con los estudiantes una rúbrica de evaluación. Los estudiantes, de forma consensuada, diseñaron para cada actividad una rúbrica en la que se reunieron los aspectos que debían ser evaluados y que por tanto debían ser retroalimentados por sus iguales. La elaboración de la rúbrica se fundamentó sobre la pregunta “¿Qué es un buen trabajo?”. A partir de esta pregunta los estudiantes eligieron y pautaron los criterios a incluir en la rúbrica de evaluación. Durante esta fase el papel de los profesores fue el de mediador. La construcción de la rúbrica fue la única fase realizada de forma presencial.

6HJXQDIDMH Administración de un pretest en el que se midió los diferentes aspectos que forman la autorregulación del aprendizaje del alumnado.

7HFHDIDUH Primera entrega de la actividad y primer feedback entre iguales. Los estudiantes entregaron una primera versión de la actividad objetivo. Esta primera versión fue revisada por sus iguales, quienes realizaron una primera corrección y feedback de acuerdo con los criterios establecidos en la rúbrica.

\&XDUD IDYH Segunda entrega de la actividad. Los estudiantes entregaron una segunda versión de la actividad en la que debían incluir el feedback de sus compañeros y la respuesta dada a este feedback, especificando qué cambios de los propuestos por sus iguales aceptaban y cuales rechazaban y porqué. 
4 XIQUDIDMH Segundo feedback entre iguales y segunda respuesta al feedback. Los estudiantes recibieron un segundo feedback sobre el cual deben trabajar, corrigiendo lo propuesto en caso de aceptarlo y justificando el rechazo de las propuestas que no son modificadas.

6H WIDMH Entrega final de la actividad. Los estudiantes entregaron la versión final de la actividad en la cual incluyeron los cambios sugeridos por sus compañeros tanto en el primer como en el segundo feedback.

$6 p S$ WPD IDMH Administración del post-test. Se realiza una segunda recogida de datos relativos a la autorregulación del aprendizaje.

2 FWYDIDH Evaluación cuantitativa y cualitativa por parte del docente. Los docentes evaluaron la versión final del trabajo emitiendo una valoración tanto cuantitativa como cualitativa.

1 RYHQD IDMH Realización de entrevistas en profundidad a 9 de los alumnos que participaron en la innovación.

' pFIP DIIDH Análisis cualitativo del tipo de feedback emitido por cada alumno.

\section{Instrumentos}

El apartado cuantitativo del estudio contó con el instrumento de medición MSLQ - SF (Zurita et al., 2019). Es una escala tipo likert de 5 puntos de administración colectiva. consta de 40 ítems que se divide en dos subescalas:

- Subescala de motivación. Enfocada a la medición de los subconstructos "tarea", "ansiedad" y "motivación intrínseca.

- Subescala de estrategias cognoscitivas. Orientada a la medición de los subconstructos "estrategias de elaboración”, "estrategias de organización”, "pensamiento crítico", "autorregulación de la metacognición", "tiempo y hábitos de estudio" y "autorregulación del esfuerzo".

El apartado cualitativo se realizó a partir de entrevistas semiestructuradas y análisis sistemático del feedback. Ambos análisis siguieron un enfoque inductivo-deductivo, por lo que contó con categorías predefinidas basadas en la literatura y categorías emergentes. El análisis realizado fue de tipo temático (Bryman, 2012).

En el caso de la entrevista, el guión se organizó en tres bloques: El primer bloque incluyó preguntas relacionadas con la construcción de la rúbrica, implementación del proceso de feedback, y evaluación del feedback como autorregulación del aprendizaje. El segundo bloque incluyó preguntas tanto del feedback que realizaron los estudiantes como el que recibieron por parte de sus compañeros/as, así como la respuesta que ofrecieron al feedback recibido. El último bloque hizo referencia a cuestiones generales sobre el proceso de aprendizaje. Para el análisis del feedback las categorías predefinidas fueron cuatro: feedback personal, feedback correctivo, feedback sobre el procesamiento de la tarea y feedback de autorregulación (Hattie y Temperley, 2007). 


\section{Resultados}

\section{Impacto de la innovación en la autorregulación del aprendizaje}

Para conocer el impacto de la innovación en la autorregulación del aprendizaje se compararon las puntuaciones post-test y pretest mediante estadísticos de comparación de medias. Se utilizaron estadísticos no paramétricos dado que los datos no mostraban una distribución normal. Los resultados de las pruebas Wilcoxon para muestras relacionadas mostraron un incremento significativo de las puntuaciones del posttest con respecto a las puntuaciones obtenidas en el pre-test en las subescalas de metas de orientación intrínseca $(\mathrm{T}=3.462 ; \mathrm{p}=.002 ; \mathrm{r}=0.250)$, autorregulación del esfuerzo $(\mathrm{T}=5.318 ; \mathrm{p}=.004 ; \mathrm{r}=0.231)$, tiempo y hábitos de estudio $(\mathrm{T}=5.284 ; \mathrm{p}=.006 ; \mathrm{r}=0.224)$, autorregulación a la metacognición $(\mathrm{T}=5.338$ $; \mathrm{p}=.019 ; \mathrm{r}=0.189)$, pensamiento crítico $(\mathrm{T}=4.486 ; \mathrm{p}=.000 ; \mathrm{r}=0.299)$ y estrategias de elaboración ( $\mathrm{T}$ $=5.310 ; \mathrm{p}=.001 ; \mathrm{r}=0.260)$ (Gráfica 1$)$.

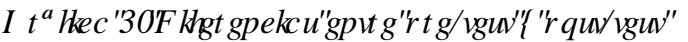

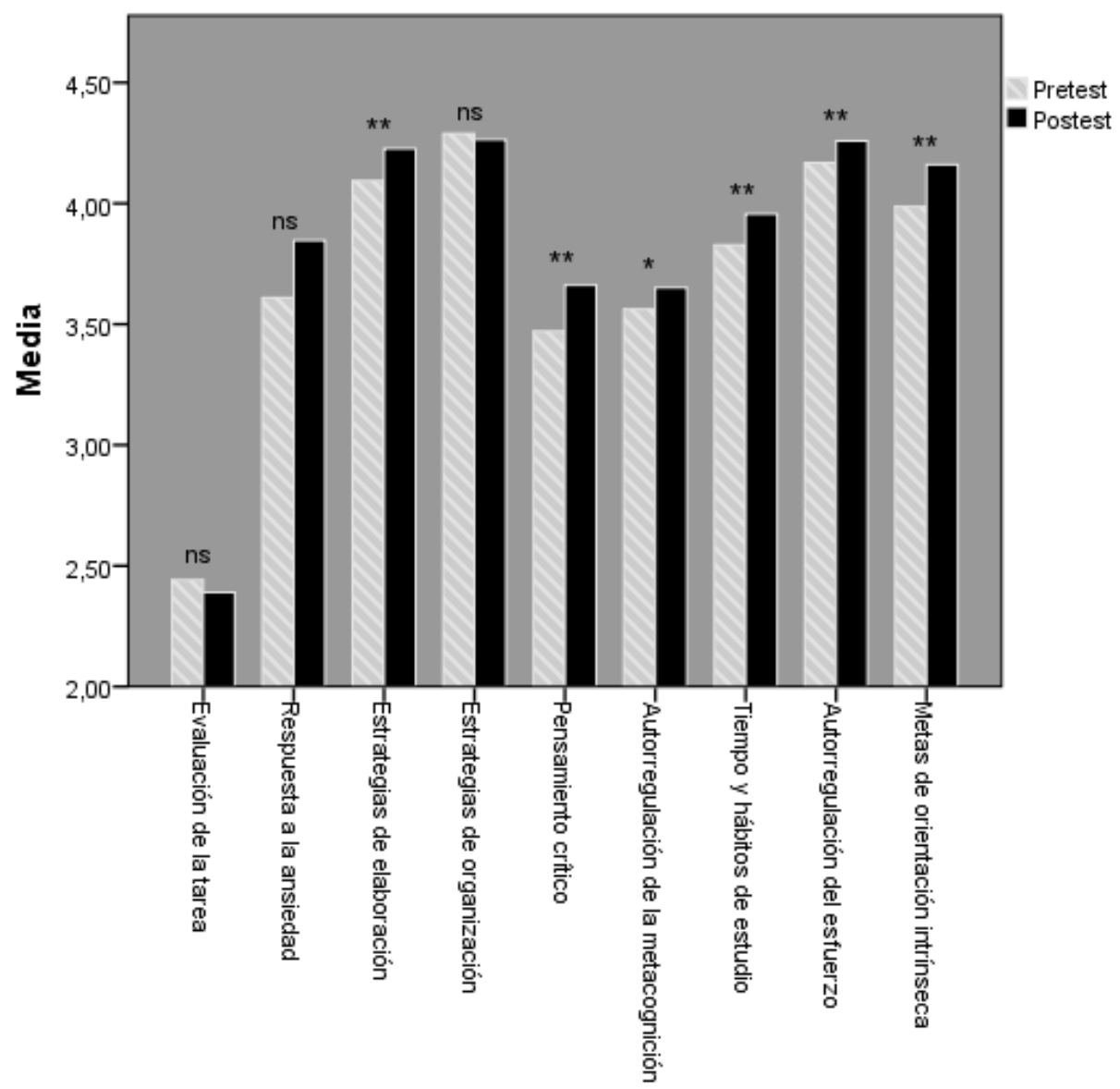




\section{Características del feedback emitido}

Para conocer las características del feedback emitido se realizó un análisis parcial basado en la clasificación de Hattie \& Temperley (2007): feedback personal, feedback correctivo, feedback del procesamiento de la tarea y feedback de autorregulación.

En el análisis se observaron matices en las categorías de feedback correctivo y de feedback de procesamiento de la tarea. La categoría de feedback correctivo se subdividió en: 1) positivo, relativo a aquellos feedbacks que califican la tarea como correcta; 2) negativo, feedback que califica la tarea como incorrecta; 3) positivo y negativo, califican la tarea positiva y negativamente de forma simultánea; y 4) parafraseo del indicador, el feedback se ciñe a parafrasear el criterio de evaluación de la rúbrica. A su vez, la categoría de feedback de procesamiento de la tarea fue subdividida en: 1) constructivo, donde el feedback explica explícitamente las estrategias que subyacen en la tarea; 2) basado en el indicador, el feedback se ciñe a la definición del indicador de evaluación; 3) basado en otro indicador, el feedback es correcto pero hace alusión a otro indicador de evaluación.

Los resultados del análisis parcial mostraron una tendencia predominante correctiva, siendo los subtipos más frecuentes el positivo y el parafraseo del indicador. En un segundo lugar, pero con una presencia significativamente menor, se encontraron los tres subtipos de feedback del procesamiento de la tarea, siendo el más escaso el de subtipo constructivo. En este análisis parcial no se ha encontrado evidencia de feedback de autorregulación.

\section{Eficacia de la innovación como herramienta para la mejora del aprendizaje virtual}

Por último, recogiendo las voces de los estudiantes a través de las entrevistas, en éstas se identificaron tres elementos que permitieron a los estudiantes desarrollar estrategias de autorregulación del aprendizaje: (1) la construcción de la rúbrica como un ejercicio de autoexigencia y de andamiaje dado que eran los mismos estudiantes quienes asignaban qué contenidos se evaluaban y a cuales se les asignaba mayor valor, (2) el uso de la rúbrica para evaluar sus propias producciones, y (3) el hecho de realizar el feedback a sus compañeros les permite reflexionar de forma constructiva y crítica sobre su propio trabajo.

Del mismo modo, las entrevistas mostraron tres motivos por los que el feedback entre iguales había resultado una herramienta útil en el proceso de evaluación en formato online: (1) facilidad de comunicación, (2) posibilidad de usar herramientas tecnológicas mientras están reunidos como, por ejemplo, realizar una videoconferencia mientras trabajan en un documento en la nube y (3) posibilidad de tener espacios virtuales de debate que reemplazan el repartirse tareas y trabajar por separado.

\section{Conclusiones}

La presente innovación propone el feedback entre iguales como medida de evaluación formativa online en el marco de la educación superior. Esta propuesta está apoyada en un estudio de tipo mixto en el que se pretende conocer, a través de un diseño longitudinal, los cambios producidos en los niveles de autorregulación del aprendizaje consecuencia del proceso de feedback, así como las experiencias en el proceso de aprendizaje del alumnado a través de una entrevista semiestructurada y sobre el efecto del tipo de feedback a través del análisis del feedback emitido.

Los resultados obtenidos en el apartado cuantitativo mostraron un incremento significativo de los valores relativos a los subconstructos "metas de orientación intrínseca, autorregulación del esfuerzo, tiempo y 
hábitos de estudio, autorregulación a la metacognición, pensamiento crítico y estrategias de elaboración. Estos resultados, en la línea del estudio de Cano y Pons (2019), sugieren que el proceso de feedback entre iguales ha permitido al alumnado desarrollar una serie de habilidades cognitivas y metacognitivas dirigidas a la dirección y modificación del propio aprendizaje.

Por su parte, a nivel cualitativo se observó cómo, en consonancia con lo observado por Abella García et al. (2020), el proceso de feedback entre iguales reportó un beneficio evidente como forma de evaluación online. Elementos dentro del ejercicio de feedback como la construcción conjunta de la rúbrica como ejercicio de autoexigencia, el uso de la rúbrica como proceso de monitorización y evaluación continua de la propia producción, y el hecho de realizar el feedback a sus compañeros como ejercicio de reflexión sobre su propio trabajo fueron particularmente importantes para explicar el éxito del proceso.

En general, los resultados obtenidos permiten proponer el proceso de feedback entre iguales como una forma de evaluación formativa capaz de resolver los problemas de adaptación de la evaluación tradicional al formato virtual.

\section{Referencias \\ [}

AJJAWI, R., y BOUD, D. (2017). "Researching feedback dialogue: An interactional analysis approach" en

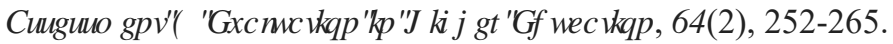

BONSÓN, M., y ÁGUEDA, B. (2005). "Evaluación y Aprendizaje. En Águeda Benito y Ana Cruz (Eds.),

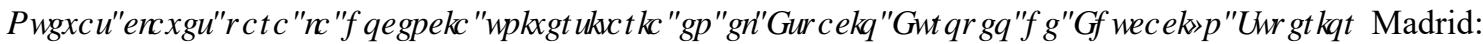
Narcea (pp. 87-100).

BRYMAN, A. (2012), 6RFDO5 HMDFKD HKRGWW UKLHGNew York: Oxford University Press.

CABAllÉ, S., DARADOUMIS, T., XHAFA, F., y JUAN, A. (2011). "Providing effective feedback, monitoring and evaluation to on-line collaborative learning discussions" en \&RPSXWWW IQ +XPDQ \%KDIRUT(4), 1372-1381.

CANO GARCÍA, E., PONS-SEGUI, L., LLUCH MOLINS, L., JARDÍ FERRÉ, A., FERNANDEZ FERRER, M., CABRERA LANZO, N., ... y PORTILLO VIDAL, C. (2020). P2P4L2L (Peer to Peer for Learning to Learn).

CANO, E., PONS-SEGUÍ, L. y LLUCH, L. (2020). ) HGEDFN HQ ( GXFDFIyQ 6XSHURU Barcelona: Universidad de Barcelona.

CARLESS, D., y CHAN, K. K. (2017). "Managing dialogic use of exemplars" en \$ WHMP HQW I YDODWRQ IQ+ LJKHU( GXFDMRQ Q (6), 930-941.

COLL, C., ROCHERA, M. J., DE GISPERT, I., y BARRIGA, F. D. (2013). "Distribution of feedback among teacher and students in online collaborative learning in small groups".en ' IJIWOO GXFDURQ5 HIIHZ, (23), 27-45.

COLL, M. F. (2016). "Tutoría entre iguales y comprensión lectora: ¿un tándem eficaz? Los efectos de la

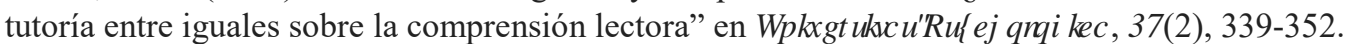

GARCÍA-PEÑALVO, F. J., ABELLA-GARCÍA, V., CORELL, A., y Grande, M. (2020). "La evaluación online en la educación superior en tiempos de la COVID-19" en ( GXFDMRQIQQWKH. QRZ GGJH6RFIHW, 21, 12.

GARCÍA-PEÑALVO, F. J., CORELL, A., RIVERO-ORTEGA, R., RODRIGUEZ-CONDE, M. J., y RODRIGUEZ-GARCÍA, N. (2021). "Impact of the COVID-19 on Higher Education: An Experience-Based

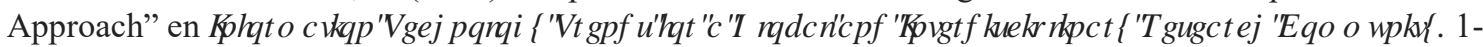
18

(c)) BY-NC-ND 2021, Universitat Politècnica de València

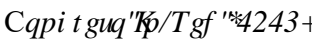


GIBBONS, S. L., y KANKKONEN, B. (2011). "Assessment as learning in physical education: Making

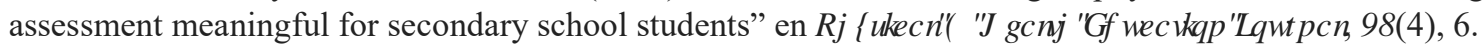

HATTIE, J., y TIMPERLEY, H. (2007). "The power of feedback" en $5 H M I H \square R I \square$ HEXFDMRQDO UMHDFK, Q(1), 81-112.

IBARRA SAIZ, M.S., RODRÍGUEZ GOMEZ, G., GÓMEZ RUIZ, M.A. (2012). "La evaluación entre

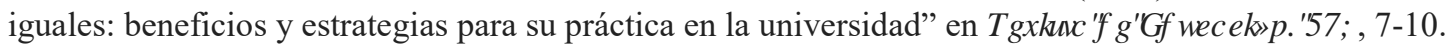

NEUGEBAUER, J., RAY, D. G., y SASSENBERG, K. (2016). "When being worse helps: The influence of upward social comparisons and knowledge awareness on learner engagement and learning in peer-to-

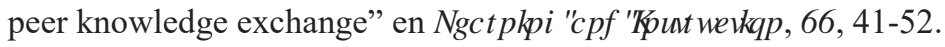

WATTS, F. y GARCÍA, A. (2006). / DHHDODFIyQFRP SDUWDIIQYHWDFFY QP XQWGLISQQDU Valencia: Universidad Politécnica de Valencia.

WILIAM, D. (2011). "What is assessment for learning?" en 6WGHMIQ( GXFDURQDO YDOXDRQ3, 3-14.

ZENG, W., HUANG, F., YU, L., y CHEN, S. (2018). "Towards a learning-oriented assessment to improve students' learning - a critical review of literature" en ( GXFDURQDO \$WHMP HQW ( IDODWRQ DQG \$FFXQUEICWMU⿴囗十丁, 211-250.

ZURITA ORTEGA, F., MARTINEZ MARTINEZ, A., CHACON CUBEROS, R., y UBAGO JIMÉNEZ, J. L. (2019). "Analysis of the Psychometric Properties of the Motivation and Strategies of Learning Questionnaire-Short Form (MSLQ-SF) in Spanish Higher Education Students" en 6RFDOGFHQFH, $\square(5)$, 132. 